\title{
Efficacy of Qingfei Paidu Decoction on Patients with COVID-19 Pneumonia in Wuhan, China: A Propensity Score Matching Study
}

\author{
Zhen Liu, ${ }^{1}$ Shan Du, ${ }^{2}$ Fei Shao, ${ }^{3,4}$ Haibin Li, ${ }^{5}$ Shuang Xu, ${ }^{6}$ Xuedi Ma, ${ }^{7}$ Zhouming Xu, ${ }^{7}$ \\ Hao Cui, ${ }^{4}$ Changxiao Yu, ${ }^{4}$ Yang Wu, ${ }^{4}$ Feng Wang, ${ }^{8,9}$ Liyan Li, ${ }^{1}$ Rui Chen, ${ }^{10}$ Hui Qiu, ${ }^{11}$ \\ Ziren Tang, ${ }^{3,4}$ and Peng Sun ${ }^{6}{ }^{6}$ \\ ${ }^{1}$ Department of Emergency Medicine, Beijing First Hospital of Integrated Chinese and Western Medicine, Beijing, China \\ ${ }^{2}$ Department of Neurology, Beijing First Hospital of Integrated Chinese and Western Medicine, Beijing, China \\ ${ }^{3}$ Department of Emergency Medicine, Beijing Chaoyang Hospital, Capital Medical University, Beijing, China \\ ${ }^{4}$ Beijing Key Laboratory of Cardiopulmonary Cerebral Resuscitation, Beijing, China \\ ${ }^{5}$ Department of Epidemiology and Health Statistics, School of Public Health, Capital Medical University, Beijing, China \\ ${ }^{6}$ Department of Emergency Medicine, Union Hospital, Tongji Medical College, Huazhong University of Science and Technology, \\ Wuhan, China \\ ${ }^{7}$ AI Research Division, A.I. Phoenix Technology Co., Ltd., Hong Kong, China \\ ${ }^{8}$ Department of Respiratory and Critical Care Medicine, Beijing Chaoyang Hospital, Capital Medical University, Beijing, China \\ ${ }^{9}$ Beijing Key Laboratory of Respiratory and Pulmonary Circulation Disorders, Beijing, China \\ ${ }^{10}$ Department of Integrated Traditional Chinese and Western Medicine, Union Hospital, Tongji Medical College, \\ Huazhong University of Science and Technology, China \\ ${ }^{11}$ Department of Emergency Surgery, the West Campus of Union Hospital, Tongji Medical College, \\ Huazhong University of Science and Technology, Wuhan, China
}

Correspondence should be addressed to Peng Sun; sp761397@sina.com

Received 11 April 2021; Accepted 28 August 2021; Published 5 October 2021

Academic Editor: Harry Lee

Copyright $\odot 2021$ Zhen Liu et al. This is an open access article distributed under the Creative Commons Attribution License, which permits unrestricted use, distribution, and reproduction in any medium, provided the original work is properly cited.

Background. In view of the global efforts to develop effective treatments for the current worldwide coronavirus 2019 (COVID-19) pandemic, Qingfei Paidu decoction (QPD), a novel traditional Chinese medicine (TCM) prescription, was formulated as an optimized combination of constituents of classic prescriptions used to treat numerous febrile and respiratory-related diseases. This prescription has been used to treat patients with COVID-19 pneumonia in Wuhan, China. Hypothesis/Purpose. We hypothesized that QPD would have beneficial effects on patients with COVID-19. We aimed to prove this hypothesis by evaluating the efficacy of QPD in patients with COVID-19 pneumonia. Methods. In this single-center, retrospective, observational study, we identified eligible participants who received a laboratory diagnosis of COVID-19 between January 15 and March 15, 2020, in the west campus of Union Hospital in Wuhan, China. QPD was supplied as an oral liquid packaged in 200-mL containers, and patients were orally administered one package twice daily 40 minutes after a meal. The primary outcome was death, which was compared between patients who did and did not receive QPD (QPD and NoQPD groups, respectively). Propensity score matching (PSM) was used to identify cohorts. Results. In total, 239 and 522 participants were enrolled in the QPD and NoQPD groups, respectively. After PSM at a 1:1 ratio, 446 patients meeting the criteria were included in the analysis with 223 in each arm. In the QPD and NoQPD groups, $7(3.2 \%)$ and $29(13.0 \%)$ patients died, and those in the QPD group had a significantly lower risk of death (hazard ratio (HR) $0.29,95 \%$ CI: $0.13-0.67$ ) than those in the NoQPD group ( $p=0.004)$. Furthermore, the survival time was significantly longer in the QPD group than in the NoQPD group $(p<0.001)$. Conclusion. The use of QPD may reduce the risk of death in patients with COVID-19 pneumonia. 


\section{Introduction}

The outbreak of the novel coronavirus pandemic has affected more than 200 countries globally, and as of March 1, 2021, over $113,467,303$ confirmed cases of coronavirus disease 2019 (COVID-19) have been reported with over 2,520,550 deaths [1]. The novel coronavirus was identified as severe acute respiratory syndrome coronavirus 2 (SARS-CoV-2), and the associated disease COVID-19 has clinical manifestations ranging from asymptomatic, mild pneumonia to serious acute respiratory distress syndrome, septic shock, and multiple organ dysfunction syndrome (MODS) [2-4].

Currently, despite supportive and symptomatic treatments, no specific medicine is recommended for the prevention or treatment of COVID-19 and many countries and organizations are racing against time to identify prophylactic and therapeutic treatments for patients [5]. On January 26, 2020, a new TCM prescription named Qingfei Paidu decoction (QPD) was formulated with expert consensus using a combination of 21 traditional Chinese medicine (TCM) ingredients based on previous clinical experience and disease data [6].

After the National Health Commission of the People's Republic of China (NHCPRC) released the QPD Diagnosis and Treatment Protocol for Novel Coronavirus Pneumonia (Trial Version 6) on February 19, 2020 [7], QPD was widely administered for the treatment of patients with COVID-19 nationally in China. Therefore, we aimed to explore the effects of QPD in patients with COVID-19 pneumonia.

\section{Methods}

2.1. Study Design. This was a single-center, retrospective, observational study comparing the clinical effectiveness of a combination of QPD and standard therapy versus standard therapy alone in the treatment of patients with COVID-19 pneumonia. The study was approved by the Ethics Committee Boards of Union Hospital, Tongji Medical College, and Huazhong University of Science and Technology, and the requirement for informed consent was waived on account of the retrospective study design.

2.2. Study Population and Setting. We identified patients who received a laboratory diagnosis of COVID-19 between January 15 and March 15, 2020, at the west campus of Union Hospital in Wuhan. The eligibility criteria were as follows: aged $\geq 14$ years and diagnosed with COVID-19 according to the interim guidelines of the World Health Organization. All enrolled inpatients had a definite outcome and received inhospital care consistent with the Diagnosis and Treatment Protocol for Novel Coronavirus Pneumonia (Trial Version 6) recommended by the NHCPRC [7].

The west campus of Union Hospital was one of the designated hospitals for patients with severe COVID-19, and 800 beds were modified as isolation wards and opened for admission to patients from January 2020. The Department of TCM was in charge of evaluating the clinical needs of patients and prescribing TCM drugs according to their symptoms and clinical features. The TCM pharmacy was provided QPD prescriptions, which were used to produce a decoction for patients in the general ward or intensive care unit (ICU).

2.3. QPD Exposure. According to the Diagnosis and Treatment Protocol for Novel Coronavirus Pneumonia (Trial Version 6) of the NHCPRC, patients with COVID-19 were cotreated with QPD and standard agents [7]. All patients in the general ward or ICU were administered QPD with the combination of Western medicine from the first day after hospital admission. Patients who met the following exclusion criteria were excluded: gastrointestinal bleeding before use of QPD, refused to take QPD, or coma patients without a feeding tube. Physicians of the Department of TCM evaluated the clinical features of the patients and prescribed QPD to those in the general ward or ICU.

The QPD was formulated as an oral liquid with each package containing $200 \mathrm{~mL}$. The patients received one package of QPD twice a day, in the morning and evening, 40 minutes after a meal. Patients with dry tongue due to fluid depletion were recommended to consume one bowl of rice soup. The amount of gypsum should be increased for patients with moderate or high fever. A treatment course was considered to be 3 days and was followed by another course of QPD if the symptoms improved but the patient had not totally recovered.

Patients with specific or other underlying diseases could be administered formulas in the subsequent course that were modified according to their actual conditions. QPD treatment was discontinued if the patient's symptoms were resolved, effects associated with contraindicated oral administration were observed, or severe adverse effects related to QPD occurred [7].

2.4. Data Collection. Information on age, sex, clinical symptoms, treatments, comorbidity, and outcome data of patients with severe COVID-19 pneumonia treated with QPD was retrieved from their electronic medical records by the physicians and entered into a database during the study period. The primary outcome was death before discharge from hospital.

2.5. Statistical Analysis. Nominal data are described using proportions, normally distributed discrete data are described as means and standard deviation (SD), and medians and interquartile ranges (IQRs) are used to represent data that were not normally distributed. Because the baseline characteristics of eligible participants differed between the two groups (Table 1), propensity score matching (PSM) was used to identify a cohort of patients with similar baseline characteristics. The propensity score was set as a conditional probability of having a particular exposure (QPD versus NoQPD) considering a set of baseline measured covariates.

The propensity score was estimated using a nonparsimonious multivariable logistic regression model with QPD as the dependent variable and all baseline 
TABLE 1: Characteristics of patients with COVID-19 pneumonia.

\begin{tabular}{|c|c|c|c|}
\hline Variables & QPD & NoQPD & SMD \\
\hline$n$ & 239 & 522 & \\
\hline \multicolumn{4}{|l|}{ Sex } \\
\hline Female $(\%)$ & $120(50.2)$ & $251(48.1)$ & 0.043 \\
\hline Age (median [IQR]) & $60.00[51.00-66.00]$ & $62.00[50.00-70.00]$ & 0.022 \\
\hline \multicolumn{4}{|l|}{ Symptoms } \\
\hline Fever & $192(80.3)$ & $439(84.1)$ & 0.099 \\
\hline Dizziness & $27(11.3)$ & $51(9.8)$ & 0.050 \\
\hline Fatigue & $128(53.6)$ & $278(53.3)$ & 0.006 \\
\hline Nausea & $35(14.6)$ & $51(9.8)$ & 0.149 \\
\hline Diarrhea & $46(19.2)$ & $100(19.2)$ & 0.002 \\
\hline Myalgia & $62(25.9)$ & $111(21.3)$ & 0.110 \\
\hline Abnormality of mentality & $9(3.8)$ & $72(13.8)$ & 0.360 \\
\hline Headache & $18(7.5)$ & $26(5.0)$ & 0.105 \\
\hline Abdominal pain & $13(5.4)$ & $20(3.8)$ & 0.077 \\
\hline Dyspnea & $179(74.9)$ & $367(70.3)$ & 0.103 \\
\hline Cough & $188(78.7)$ & $395(75.7)$ & 0.071 \\
\hline Sputum & $105(43.9)$ & $214(41.0)$ & 0.059 \\
\hline Chest pain & $14(5.9)$ & $22(4.2)$ & 0.075 \\
\hline \multicolumn{4}{|l|}{ Comorbidity } \\
\hline Hypertension & $45(18.8)$ & $105(20.1)$ & 0.032 \\
\hline Diabetes & $21(8.8)$ & $18(3.4)$ & 0.224 \\
\hline Coronary heart disease & $13(5.4)$ & $36(6.9)$ & 0.061 \\
\hline COPD & $1(0.4)$ & $3(0.6)$ & 0.022 \\
\hline Cancer & $6(2.5)$ & $14(2.7)$ & 0.011 \\
\hline Chronic renal disease & $0(0.0)$ & $2(0.4)$ & 0.088 \\
\hline Cerebrovascular disease & $5(2.1)$ & $12(2.3)$ & 0.014 \\
\hline
\end{tabular}

SMD $>0.1$ indicates a difference in baseline characteristics between both groups. QPD, Qingfei Paidu decoction; SMD, standardized mean difference; IQR, interquartile range; COPD, chronic obstructive pulmonary disease.

characteristics outlined in Table 1 as covariates. Matching was performed using a $1: 1$ matching protocol without replacement (greedy matching algorithm) with caliper width $=0.2$ times the SD of the propensity score logit. Absolute standardized mean differences of less than $10.0 \%$ for a given covariate indicate a relatively small imbalance.

Patient data were analyzed from baseline to the date of death or discharge, and the primary outcome was based on the PSM. Kaplan-Meier survival curves and stratified logrank tests were used, and the hazard ratio (HR) and corresponding 95\% confidence intervals (CIs) were calculated using the Cox proportional hazard models.

Analyses were performed using $\mathrm{R}$ version 3.6.3, all tests were two-sided, and $p$ values $<0.05$ were considered statistically significant.

\section{Results}

From January 15 to March 15, 2020, 792 inpatients with COVID-19 were assessed for eligibility. Thirty-one patients were excluded, with 4 not meeting the inclusion criteria and 27 missing data (Figure 1). In total, we identified 761 eligible participants, comprising 239 and 522 who were treated with and without QPD, respectively, and the patient characteristics are shown in Table 1. In the unmatched comparison of baseline characteristics of patients, no differences were observed between the QPD and NoQPD groups in sex and age. Among the variables analyzed, symptoms of nausea, myalgia, mental abnormalities, and diabetes as a comorbidity were significantly different between the two groups (Table 1).

After PSM at a $1: 1$ ratio, 446 patients meeting the criteria were included in the analysis with 223 patients in each arm. The mean age of the QPD and NoQPD groups was 59 and 61 years, and $50.2 \%$ and $49.3 \%$ of the participants were women, respectively (Table 2). Baseline demographic characteristics, symptoms, and comorbidities were almost comparable between the two groups except for the history of diabetes (Table 2). Furthermore, 7 (3.2\%) and 29 (13.0\%) patients died in the QPD and NoQPD groups, respectively.

Patients in the QPD group had a significantly $(p=0.004)$ lower risk of death (HR 0.29, 95\% CI: 0.13-0.67) than those in the NoQPD group. We also conducted a sensitivity analysis, and after adjusting for baseline age, sex, and diabetes, patients in the QPD group also showed a significantly ( $p=0.004$ ) lower risk of death (HR 0.30, 95\% CI: 0.13-0.68) than those in the NoQPD group (Table 3). Figure 2 shows the Kaplan-Meier survival curves.

\section{Discussion}

This retrospective analysis showed that cotreatment of patients with COVID-19 pneumonia with QPD and conventional treatment was associated with higher rates of survival and hospital discharge, lower death rates, and longer survival time than conventional treatment alone. Following the 


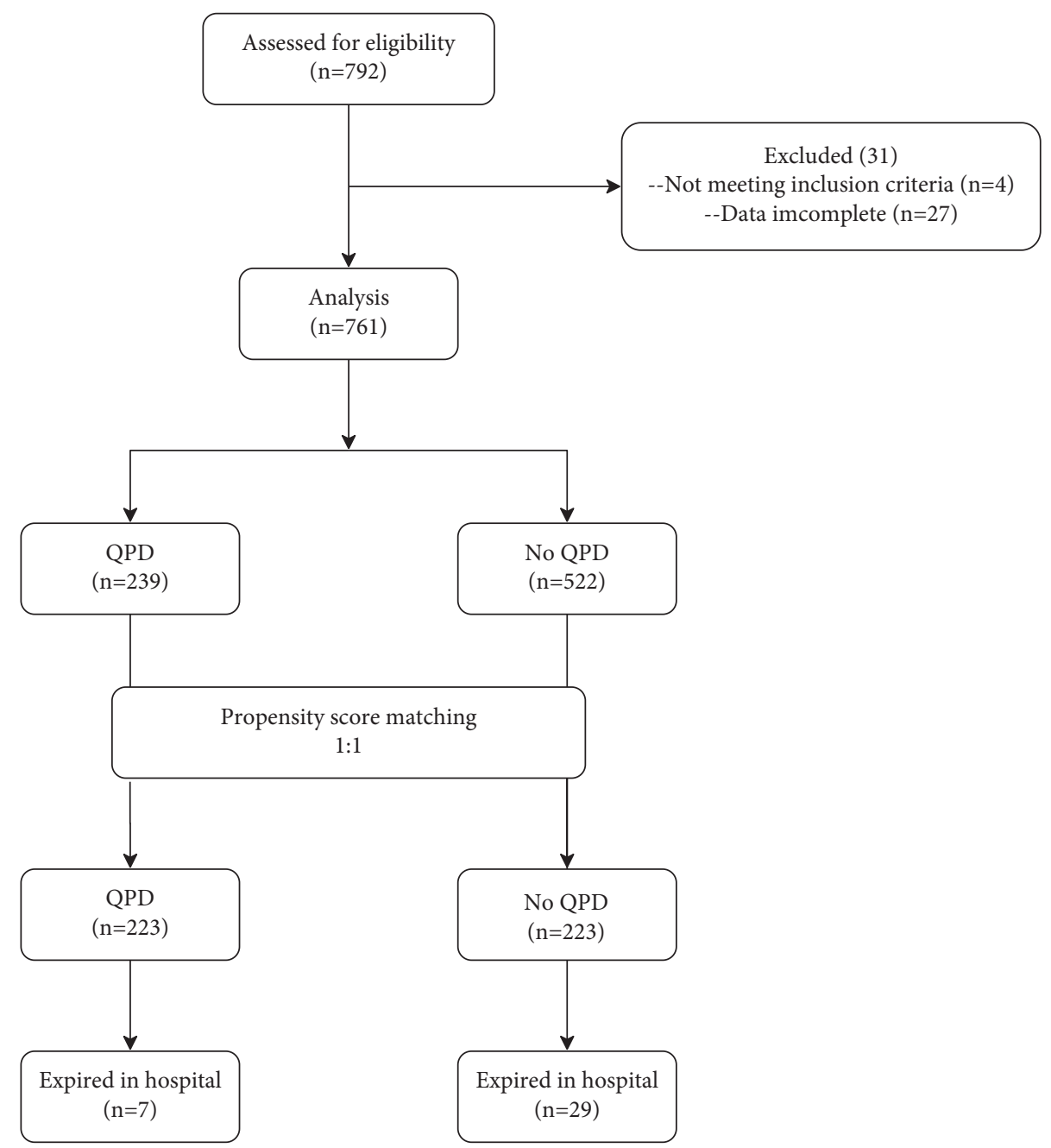

FIGURE 1: Flow diagram illustrating the number of patients during the study.

outbreak of COVID-19, timely intervention using TCM has played an important role in its treatment in China. The NHCPRC issued seven versions of its diagnosis and treatment protocol for COVID-19 with recommendations to combine TCM prescriptions and standard treatment since the third version was issued on January 23, 2020 [7-9].

TCM is a complete theoretical system that has played an indispensable role in the prevention and treatment of several epidemic diseases in China over the years [10]. For instance, in 2003, TCM prescriptions were used to prevent and treat SARS. TCM herbal extracts of Cibotium barometz, Gentiana scabra, Dioscorea batatas, Cassia tora, and Taxillus chinensis can inhibit SARS-CoV replication [11]. During the influenza A virus subtype H1N1 (H1N1) influenza pandemic of 2009, oseltamivir and maxingshigan-yinqiaosan alone and in combination with standard agents reduced time to fever resolution in infected patients. Consequently, maxingshigan-yinqiaosan may be an alternative treatment for H1N1 influenza virus infection [12].

QPD is an optimized combination of classic prescriptions used for the treatment of multiple exogenous febrile diseases. It is composed of 21 herbs (Table 4) derived from Maxing Shigan decoction (MSD), Shegan Mahuang decoction (SMD), Xiaochaihu decoction (XCHD), and Wuling powder in the "Treatise on Febrile and Miscellaneous Diseases" by Dr. Zhang Zhongjing in the Han Dynasty. Among these constituents, the main active ingredient is MSD, which consists of Herba Ephedrae, Semen armeniacae amarum, Gypsum fibrosum, and Radix Glycyrrhizae.

MSD has been widely used in the prevention and treatment of respiratory diseases. Several studies had verified its antiviral effects mediated by regulating immune function, suppressing inflammatory cytokine storm, and improving oxygenation and forced expiratory volume/second (FEV1) $[13,14]$. SMD has been shown to alleviate pathological lung damage and inflammation in rats with asthmatic pneumonia by enhancing immune activity and downregulating the expression of thymic stromal lymphopoietin (TSLP), tolllike receptor 4 (TLR4), and nuclear factor $-\kappa \mathrm{B}(\mathrm{NF}-\kappa \mathrm{B})$ in lung tissue [15].

$\mathrm{XCHD}$ has immunomodulatory, anti-liver injury, and anti-inflammatory effects mediated by regulating the level of pathogens on the half-superficies and half-interior and inhibiting the inflammatory cytokines tumor necrosis factor (TNF- $\alpha$ ), IL-1 $\beta$, IL-6, and macrophage colonystimulating factor (M-CSF) [16, 17]. Wuling powder 
TABle 2: Characteristics of patients with COVID-19 pneumonia after propensity score matching (1:1 match).

\begin{tabular}{lccc}
\hline Variables & QPD & NoQPD & SMD \\
\hline \multicolumn{1}{c}{$n$} & 223 & 223 & \\
\hline Sex & & & \\
Female (\%) & $112(50.2)$ & $110(49.3)$ & 0.018 \\
\hline Age (median [IQR]) & 59.00 & 61.00 & \\
& {$[51.00-66.00]$} & {$[49.00-69.00]$} & 0.001 \\
\hline Symptoms & & & \\
Fever & $179(80.3)$ & $184(82.5)$ & 0.058 \\
Dizziness & $24(10.8)$ & $25(11.2)$ & 0.014 \\
Fatigue & $119(53.4)$ & $121(54.3)$ & 0.018 \\
Nausea & $33(14.8)$ & $35(15.7)$ & 0.025 \\
Diarrhea & $43(19.3)$ & $45(20.2)$ & 0.023 \\
Myalgia & $56(25.1)$ & $62(27.8)$ & 0.061 \\
Abnormality of & $9(4.0)$ & $8(3.6)$ & 0.023 \\
mentality & $18(8.1)$ & $15(6.7)$ & 0.051 \\
Headache & $13(5.8)$ & $12(5.4)$ & 0.019 \\
Abdominal pain & $164(73.5)$ & $164(73.5)$ & $<0.001$ \\
Dyspnea & $174(78.0)$ & $169(75.8)$ & 0.053 \\
Cough & $100(44.8)$ & $90(40.4)$ & 0.091 \\
Sputum & $14(6.3)$ & $10(4.5)$ & 0.080 \\
Chest pain & & & \\
\hline Comorbidity & $43(19.3)$ & $39(17.5)$ & 0.046 \\
Hypertension & $17(7.6)$ & $10(4.5)$ & 0.132 \\
Diabetes & $11(4.9)$ & $14(6.3)$ & 0.059 \\
Coronary heart & $1(0.4)$ & $1(0.4)$ & $<0.001$ \\
disease & $6(2.7)$ & $6(2.7)$ & $<0.001$ \\
COPD & $0(0.0)$ & $0(0.0)$ & $<0.001$ \\
Cancer & $5(2.2)$ & $5(2.2)$ & $<0.001$ \\
Chronic renal & disease & & \\
Cerebrovascular & disease & & \\
\hline
\end{tabular}

QPD, Qingfei Paidu decoction; SMD, standardized mean difference; IQR, interquartile range; COPD, chronic obstructive pulmonary disease. All variables except diabetes had SMD values less than 0.1, indicating that the two groups were comparable.

possesses strong anti-inflammatory activity and acts through various signaling pathways, including $\mathrm{NF}-\kappa \mathrm{B}$, mitogen-activated protein kinases (MAPKs), and heme oxygenase-1 (HO-1) [18].

A pharmacokinetic study has shown that the potent inhibition of CYP1A and CYP3A by QPD partially blocks the formation of the oxidative metabolites of arachidonic acid, thereby alleviating systemic inflammation in patients with COVID-19. Thus, QPD may exert its anti-inflammatory effects by inhibiting human P450s [19]. Some core components of QPD have an affinity for the novel SARSCoV-2 main protease (3C-like protease, 3CLpro) and angiotensin-converting enzyme 2 (ACE2) [20]. Furthermore, by regulating a series of proteins coexpressed with ACE2 and signaling pathways closely related to the occurrence and development of diseases, it plays a role in balancing immunity and eliminating inflammation. It may act as an antiviral agent by targeting ribosomal proteins that are necessary for viral replication, thereby inhibiting viral mRNA translation and group of proteins that interact with viral proteins [21].
As indicated in the sixth edition of the recommendation guidelines, QPD is suitable for mild, moderate, and severe cases of COVID-19 in accordance with clinical observations of doctors in various locations and can be used reasonably after considering the actual conditions of critically ill patients [7]. Under the guidance, patients should be administered QPD with appropriately adjusted constituents according to their specific conditions, such as body temperature, diet, and symptoms.

Studies have shown that the combination of QPD and conventional treatment relieves the symptoms of COVID19 and resolves inflammation in the lung [22]. Early treatment with QPD may serve as an effective strategy in controlling the pandemic, as early treatment with QPD is associated with favorable outcomes, including faster recovery, shorter time to viral shedding, and shorter duration of hospital stay [23]. Although the mechanism of the pharmacological action of QPD in the treatment of COVID-19 is complex, its primary site of action is the lung, which indicates that the decoction has specific effects against lung diseases [24]. Moreover, two studies with small samples have suggested that QPD combined with Western medicine in the treatment of COVID-19 is more effective than western medicine alone and can significantly shorten the patient's hospitalization time, clinical symptom improvement time, and lung CT improvement time; however, neither mortality nor length of hospitalization was affected [25].

QPD includes ephedra, which is banned in the US due to toxicity and is not considered safe by the EFSA. The ban on ephedra-containing supplements continues to be controversial. Ephedra is legal and widely used in several countries, such as Germany, Japan, India, and China. According to the Chinese Pharmacopoeia, Herba Ephedrae (ma huang) has a total alkaloid content of $1 \%$ by dry weight, and honey-frying decreases the alkaloid content by $0.194 \%$. The safe daily dose of Herba Ephedrae is 2-9 g/d. QPD contains $9 \mathrm{~g}$ of Herba Ephedrae and caused no serious adverse effects.

Our study found that the administration of QPD reduced the mortality rate of patients with COVID-19 and for the British and Indian COVID-19 variants that are currently emerging, Traditional Chinese medicine may be an option. In the future, high-quality, prospective, controlled studies are required to confirm the efficacy and the safety; meanwhile, basic research is also needed to explore the mechanisms of TCM prescriptions to provide new approaches for the treatment of COVID-19.

4.1. Limitations. Notably, there are some limitations to this study. First, although we used PSM to eliminate the bias caused by confounders, unmeasured and residual confounding factors could still have influenced our results. For example, the timing of administration and sequence in relation to other interventions, severity of illness among patients, or a combination of these factors may have differed between those treated with and without QPD. Second, some risk factors, such as D-dimer, sequential organ failure assessment score, and lactate dehydrogenase $[4,26]$, were not 
TABLE 3: Outcome event analysis of samples based on propensity score matching.

\begin{tabular}{|c|c|c|c|c|c|}
\hline & Death rate & HR $(95 \% \mathrm{CI})^{*}$ & $p$ value & HR $(95 \% \mathrm{CI})^{\#}$ & $\overline{p \text { value }}$ \\
\hline NoQPD $(n=223)$ & $29(13.0 \%)$ & 1.00 & & 1.00 & \\
\hline QPD $(n=223)$ & $7(3.2 \%)$ & $0.29[0.13-0.67]$ & 0.004 & $0.30[0.13-0.68]$ & 0.004 \\
\hline
\end{tabular}

QPD, Qingfei Paidu decoction; HR, hazard ratio. * Unadjusted. "Adjusted for baseline age, sex, and diabetes.
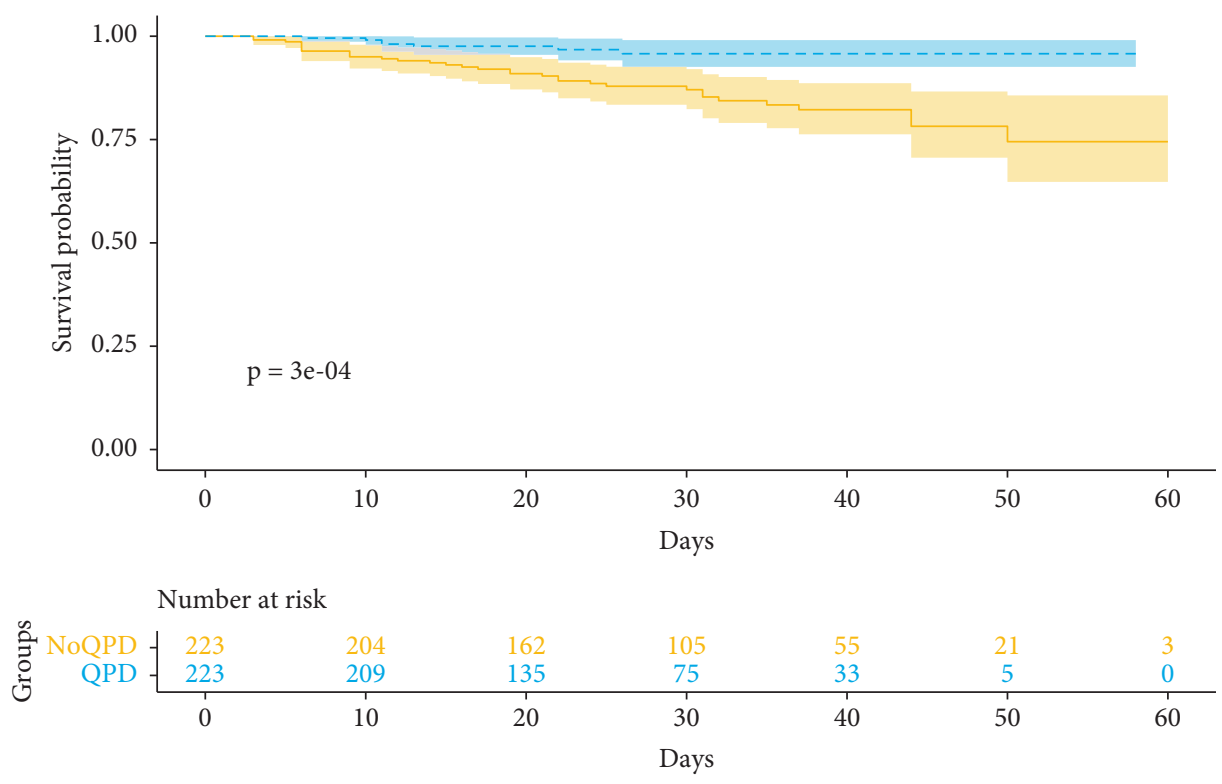

Groups

NoQPD

QPD

FIGURE 2: Survival curve by treatment group.

TABle 4: Ingredient list of the Qingfei Paidu decoction.

\begin{tabular}{lccc}
\hline & Ingredients in Chinese & Ingredients in English & Ingredients in Latin \\
\hline 1 & Ma huang & Ephedra herb & Herba ephedrae \\
2 & Gan cao, baked & Liquorice root & Radix glycyrrhizae \\
3 & Ku xing ren & Bitter apricot seed & Demen armeniacae amarum \\
4 & Sheng shi gao & Gypsum & Gypsum fibrosum \\
5 & Gui zhi & Cassia twig & Ramulus cinmomi \\
6 & Ze xie & Oriental waterplantain rhizome & Rhizoma alismatis \\
7 & Zhu ling & Agaric & Polyporus umbellatus \\
8 & Bai zhu & Largehead atractylodes rhizome & Atractylodis macrocephalae rhizoma \\
9 & Fu ling & Tuckahoe & Poria \\
10 & Chai hu & Chinese thorowax root & 9 \\
11 & Huang qin & Baical skullcap root & Stellariae radix \\
12 & Jiang ban xia & Ginger processed pinellia & Radix scutellariae \\
13 & Sheng jiang & Common ginger & Pinelliae rhizoma praeparatum cum zingibere \\
14 & Zi wan & Tatarian aster root & Rhizoma zingiberis recens \\
15 & Kuan dong hua & Common coltsfoot flower & Radix asteris \\
16 & She Gan & Blackberry lily rhizome & Flos farfarae \\
17 & Xi xin & Manchurian wildginger & 9 \\
18 & Shan yao & Common yam rhizome & 9 \\
19 & Zhi shi & Immature bitter orange & Rhizoma belamcandae \\
20 & Chen pi & Tangerine peel & Herba asari \\
21 & Guang huo xiang & Benth cablin patchouli herb & Rhizoma dioscoreae \\
\hline & & & Fructus aurantii immaturus
\end{tabular}


included in our analysis. Third, there are few pharmacokinetic studies on QPD because it is a new formulation developed during the recent COVID-19 outbreak. Further studies to explore the mechanisms of action of QPD are needed. Lastly, other TCM formulations were administered to patients in the study and we could not specify their effects, which could have affected the result of this study.

\section{Conclusions}

QPD may reduce the risk of death in patients with COVID19. Further randomized controlled clinical trials are needed to corroborate these findings and provide definite conclusions. Finally, pharmacokinetic studies on QPD and its expanded use are needed in the future.

\section{Abbreviations}

COVID-19: Coronavirus disease 2019

PSM: $\quad$ Propensity score matching

QPD: $\quad$ Qingfei Paidu decoction

TCM: $\quad$ Traditional Chinese medicine.

\section{Data Availability}

The datasets used and/or analyzed during the current study are available from the corresponding author on reasonable request.

\section{Disclosure}

The funding sources were not involved in the study design, collection, analysis, and interpretation of data, or the decision to submit the manuscript for publication.

\section{Conflicts of Interest}

The authors declare that they have no conflicts of interest.

\section{Authors' Contributions}

Zhen Liu and Shan Du contributed equally to this work; Ziren Tang and Peng Sun contributed equally to this work.

\section{Acknowledgments}

The authors thank their colleagues, Mei Yang and Jianbin Tang, who collected and computed the medical information of the patients included in this study. The authors thank the patients and their families for making this study possible. The authors also thank all the healthcare providers of Union Hospital, as well as the supporting teams from 12 provinces for resuscitation efforts. The authors would also like to express their appreciation to other colleagues of A.I. Phoenix Technology Co., Ltd, Jiayou Lyu, Peng Zhao, Jiwen You, Yuwei Liu, Shihao Wang, and Yunfei Tang, who contributed considerably to the process of data cleaning and analysis. This study was supported by the National Nature Science Foundation of China (Grant no. 81571866).

\section{References}

[1] World Health Organization, Weekly Operational Update on COVID-19, 2021, https://www.who.int/publications/m/item/ weekly-operational-update-on-covid-19---1-march-2021.

[2] T. Chen, D. Wu, H. Chen et al., "Clinical characteristics of 113 deceased patients with coronavirus disease 2019: retrospective study," BMJ, p. m1091, 2020.

[3] D. Wang, Y. Yin, C. Hu et al., "Clinical course and outcome of 107 patients infected with the novel coronavirus, SARS-CoV2, discharged from two hospitals in Wuhan, China," Critical Care (London, England), vol. 24, no. 1, p. 188, 2020.

[4] X. Yang, Y. Yu, J. Xu et al., "Clinical course and outcomes of critically ill patients with SARS-CoV-2 pneumonia in Wuhan, China: a single-centered, retrospective, observational study," The Lancet Respiratory Medicine, vol. 8, no. 5, pp. 475-481, 2020.

[5] World Health Organization, QঊA on Coronaviruses, https://www.who.int/news-room/q-a-detail/q-acoronaviruses.

[6] National Administration of Traditional Chinese Medicine, Notice on Recommending the Use of" QPD "in the Treatment of COVID-19 with Integrated Chinese and Western Medicine, 2020, http://yzs.satcm.gov.cn/zhengcewenjian/2020-02-07/12 876.html.

[7] National Health Commission, PRC, "Diagnosis and treatment protocol for novel coronavirus pneumonia (Trial Version 6)," 2020.

[8] National Health Commission, PRC, "Diagnosis and treatment protocol for novel coronavirus pneumonia (Trial Version 3)," 2020.

[9] National Health Commission, PRC, "Diagnosis and treatment protocol for novel coronavirus pneumonia (Trial Version 7)," Chinese Medical Journal, vol. 133, pp. 1087-1095, 2020.

[10] J.-1. Ren, A.-H. Zhang, and X.-J. Wang, "Traditional Chinese medicine for COVID-19 treatment," Pharmacological Research, vol. 155, Article ID 104743, 2020.

[11] C.-C. Wen, L.-F. Shyur, J.-T. Jan et al., "Traditional Chinese medicine herbal extracts of Cibotium barometz, Gentiana scabra, Dioscorea batatas, Cassia tora, and Taxillus chinensis inhibit SARS-CoV replication," Journal of traditional and complementary medicine, vol. 1, no. 1, pp. 41-50, 2011.

[12] C. Wang, B. Cao, Q.-Q. Liu et al., "Oseltamivir compared with the Chinese traditional therapy maxingshigan-yinqiaosan in the treatment of H1N1 influenza: a randomized trial," Annals of Internal Medicine, vol. 155, no. 4, pp. 217-225, 2011.

[13] C.-F. Hsieh, C.-w. Lo, C.-H. Liu et al., "Mechanism by which ma-xing-shi-gan-tang inhibits the entry of influenza virus," Journal of Ethnopharmacology, vol. 143, no. 1, pp. 57-67, 2012.

[14] S. Liu, J. Chen, J. Zuo, J Lai, L Wu, and X Guo, "Comparative effectiveness of six Chinese herb formulas for acute exacerbation of chronic obstructive pulmonary disease: a systematic review and network meta-analysis," BMC Complementary and Alternative Medicine, vol. 19, no. 1, p. 226, 2019.

[15] H. Chen, J. Chen, X. B. Shen, and K. J. Lyu, "Effect of Shegan Mahuang decoction on pulmonary inflammatory response and immune response in rats with asthmatic pneumonia," Journal of Guangzhou University of Traditional Chinese Medicine, vol. 37, no. 02, pp. 317-323, 2020.

[16] L. M. Wang, J. Yu, and L. Zhang, "Experimental study on effect of Xiaochaihu Decoction on serum cytokines TNF- $\alpha$, IL-1 $\beta$, IL-6 and M-CSF in CFA Rats," Chinese Archives of Traditional Chinese Medicine, vol. 22, no. 08, p. 133, 2020. 
[17] Y. Zhang and X. Y. Zhou, "The influence on immune system and clinical application of bupleuri decoction," Guiding Journal of Traditional Chinese Medicine and Pharmacy, vol. 22, no. 08 , pp. 116-118, 2016.

[18] Y.-C. Oh, Y. H. Jeong, J.-H. Ha, W.-K. Cho, and J. Y. Ma, "Oryeongsan inhibits LPS-induced production of inflammatory mediators via blockade of the NF-kappaB, MAPK pathways and leads to HO-1 induction in macrophage cells," BMC Complementary and Alternative Medicine, vol. 14, no. 1, p. 242, 2014.

[19] F. Zhang, J. Huang, W. Liu et al., "Inhibition of drug-metabolizing enzymes by Qingfei Paidu decoction: implication of herb-drug interactions in COVID-19 pharmacotherapy," Food and Chemical Toxicology, vol. 149, p. 111998, 2021.

[20] H. Wu, J. Q. Wang, and Y. W. Yang, "Preliminary exploration of the mechanism of Qingfei Paidu decoction against novel coronavirus pneumonia based on network pharmacology and molecular docking technology," Acta Pharmaceutica Sinica, vol. 55, no. 03, pp. 374-383, 2020.

[21] J. Zhao, S. S. Tian, and J. Yang, "Investigating mechanism of Qing-Fei-Pai-Du-Tang for treatment of COVID-19 by network pharmacology," Chinese Traditional and Herbal Drugs, vol. 51, no. 04, pp. 829-835, 2020.

[22] S. Xin, X. Cheng, B. Zhu et al., "Clinical retrospective study on the efficacy of Qingfei Paidu decoction combined with Western medicine for COVID-19 treatment," Biomedicine \& Pharmacotherapy, vol. 129, Article ID 110500, 2020.

[23] N. Shi, B. Liu, N. Liang et al., "Association between early treatment with Qingfei Paidu decoction and favorable clinical outcomes in patients with COVID-19: a retrospective multicenter cohort study," Pharmacological Research, vol. 161, p. 105290, 2020.

[24] Z. H. Tian, J. J. Xiang, and J. Ge, "Novel coronavirus pneumonia treated by Qingfei Paidu decoction: theoretical analysis and clinical practice," World Chinese Medicine, vol. 15, no. 04, pp. 497-501, 2020.

[25] X.-Y. Hu, Y. Wang, J. Chen, T. Greenhalgh, and J. Wardle, "Chinese herbal medicine (" 3 medicines and 3 formulations") for COVID-19: rapid systematic review and meta-analysis," Research Square, 2021.

[26] F. Zhou, T. Yu, R. Du et al., "Clinical course and risk factors for mortality of adult inpatients with COVID-19 in Wuhan, China: a retrospective cohort study," The Lancet, vol. 395, no. 10229, pp. 1054-1062, 2020. 